\title{
Primary Ciliary Dyskinesia: Ancestral Haplotypes Analysis of the RSPH4A Founder Mutation in Puerto Rico
}

Wilfredo De Jesús-Rojas 1, 2, 3 , Dalilah Reyes De Jesús ${ }^{1}$, Angélica M. Nieves ${ }^{4}$, Ricardo A. Mosquera ${ }^{5}$, Juan C. Martinez-Cruzado ${ }^{6}$

1. Department of Pediatrics, University of Puerto Rico, Medical Sciences Campus, San Juan, PRI 2. Department of Pediatrics, Ponce Health Sciences University, Ponce, PRI 3. Department of Pediatrics, San Juan Bautista Medical School, Caguas, PRI 4. School of Medicine, Ponce Health Sciences University, Ponce, PRI 5. Division of Pediatric Pulmonology, University of Texas (UT) Physicians High Risk Children's Clinic at McGovern Medical School at UTHealth, Houston, Texas, USA 6. Department of Biology, University of Puerto Rico in Mayagüez, Mayagüez, PRI

Corresponding author: Wilfredo De Jesús-Rojas , wilfredo.dejesus3@upr.edu

\begin{abstract}
Genetic mutations in $>50$ genes, including $R S P H 4 A$, can lead to primary ciliary dyskinesia (PCD). RSPH4A mutations affect radial spokes, which alter the configuration of the ciliary ultrastructure and lead to chronic oto-sinopulmonary disease. The RSPH4A [c.921+3_6delAAGT] founder mutation was described as one cause of PCD without laterality defects in Puerto Rico. The average Puerto Rican genetic composition includes 64\% European, 21\% African ancestral, and 15\% Native-American or Taino, a native tribe in the Caribbean at the start of the European colonization, genes. Due to the relatively elevated Taino ancestry on the island, it might have contributed to the endemicity of the RSPH4A [c.921+3_6delAAGT] splice site mutation. However, the ancestry of this mutation is still not confirmed. This article describes the two pediatric PCD cases with the Puerto Rican founder mutation and reports an ancestral haplotype analysis of the RSPH4A [c.921+3_6delAAGT] splice site mutation. A median-joining haplotype network was constructed with the genome sequence data from 104 Puerto Rican subjects in the 1000 Genomes Project (1000GP). This study found that the RSPH4A [c.921+3_6delAAGT] splice site mutation was carried to Puerto Rico from Europe by conquistadors or shortly after the conquest and that it gained frequency on the island through genetic drift fueled by a subsequent population expansion.
\end{abstract}

Review began 08/21/2021 Review ended 08/28/2021 Published 09/03/2021

\section{(c) Copyright 2021}

De Jesús-Rojas et al. This is an open access article distributed under the terms of the Creative Commons Attribution License CC-BY 4.0., which permits unrestricted use, distribution, and reproduction in any medium, provided the original author and source are credited.

Categories: Genetics, Pediatrics, Pulmonology

Keywords: primary ciliary dyskinesia, rsph4a, ancestry, founder mutation, puerto rico

\section{Introduction}

Primary ciliary dyskinesia (PCD) is a genetically heterogeneous autosomal recessive disorder characterized by motile cilia dysfunction, which affects approximately one in 15,000 individuals in the United States [1]. Genetic mutations in $>50$ human genes can lead to PCD, affecting the function of many ciliary structural proteins [2-3]. These abnormalities result phenotypically in neonatal respiratory distress in approximately $80 \%$ despite a full-term gestation and chronic oto-sinopulmonary disease since birth, as well as male infertility and organ laterality identified later in life in 50\% of cases [4]. New guidelines for the diagnosis of PCD have included genetic testing as part of the diagnostic algorithm [5-6]. Several PCD mutations have been discovered as genetic testing has become more accessible to physicians, including mutations affecting the RSPH4A gene [7]. RSPH4A mutations located in chromosome six affect cilia radial spokes, which alters the configuration and function of the ciliary ultrastructure [8-9]. Previous studies have described several cases of PCD without laterality defects in individuals with Puerto Rican heritage and native Puerto Ricans due to the RSPH4A [c.921+3_6delAAGT] (rs869320683) splice site mutation [7,10].

The average Puerto Rican genetic composition includes 64\% European, 21\% African, and 15\% NativeAmerican or Taino ancestral genes [11]. Due to the endemicity of the mutation and the relatively elevated Taino ancestry on the island, RSPH4A [c.921+3_6delAAGT] splice site mutation has been described as likely being of Taino origin, the main Native American tribe in the Caribbean at the start of the European colonization [7,12]. However, no previous studies have been completed to explore the ancestry of the RSPH4A [c.921+3_6delAAGT] splice site mutation. We present a compound heterozygous and a homozygous pediatric case for the Puerto Rican founder RSPH4A [c.921+3_6delAAGT] splice site mutation. Also, we report the ancestral haplotype analysis for the Puerto Rican founder mutation.

Preliminary data of this article were previously presented as a meeting abstract at the 2020 American Thoracic Society Annual Scientific Meeting Am J Respir Crit Care Med 2020;201:A5328. Internet address: https://www.atsjournals.org/doi/pdf/10.1164/ajrccm-conference.2020.201.1_MeetingAbstracts.A5328

\section{Case Presentation}




\section{Case 1: RSPH4A compound heterozygous male pediatric case}

This was a case of an 11-year-old native Puerto Rican male with a past medical history of neonatal respiratory distress despite term gestation, daily wet-cough, and recurrent oto-sinopulmonary infections. The patient was diagnosed with pneumonia at two days old and admitted to the neonatal intensive care unit (NICU) where he stayed for 14 days on positive pressure without endotracheal intubation. The patient had multiple hospitalizations through his first three years of life due to bronchiolitis and pneumonia and was diagnosed with asthma at four years old. Adenoidectomy and tonsillectomy were done at six years old and myringotomy and three times afterward. Physical examination was pertinent for bilateral nasal polyps, bibasilar crackles, and mild generalized clubbing. Chest imaging showed persistent chronic right middle lobe (RML) and left lower lobe (LLL) atelectasis, and high-resolution computer tomography (HRCT) of the chest showed bilateral cylindrical and varicose bronchiectasis at RML and LLL. Ciliary biopsy was positive for an abnormal number and distribution of microtubules $[7+2,9+0]$ with central pair defects. Spirometry resulted in forced vital capacity (FVC): 78\%, Forced expiratory volume in the first second (FEV1): 71\%, FEV1/FVC: $91 \%$, total leucocyte count (TLC): $89 \%$, residual volume (RV): 168\%, RV/TLC: $177 \%$ percentage of predicted, consistent with a pseudo-restrictive pattern and presence of air trapping. Bronchoscopy showed normal airway anatomy and normal segmental distribution. Abundant thick and greenish secretions bilaterally were observed mostly in the RML and LLL. Bronchoalveolar lavage fluid resulted positive for Pseudomonas aeruginosa pulmonary infection. The sweat test was in $32.3 \mathrm{mEq} / \mathrm{L}$ on the right arm and $35.6 \mathrm{mEq} / \mathrm{L}$ on the left arm. Genetic testing results were negative for cystic fibrosis transmembrane conductance regulator (CFTR) genetic mutations. Serum immunoglobulin titers were normal, except for an elevated immunoglobulin E of $902 \mathrm{UI} / \mathrm{mL}$. The genetic panel for primary immunodeficiencies showed a heterozygous variant of unknown significance (VUS) at the CTC1 gene c.2803C >T (p.Leu935Phe). Fractional exhaled nitric oxide (FeNO) was measured, resulting in $<5 \mathrm{ppm}$. Nasal nitric oxide (nNO) was unavailable. Genetic sequence analysis and deletion/duplication for 36 PCD genes was completed and revealed one pathogenic variant at the $R S P H 4 A$ gene $\left[c .921+3 \_921+6\right.$ delAAGT] splice site mutation. Additionally, one likely pathogenic variant was present: $R S P H 4 A$ [c.1103T>G (p.Val368Gly)] and a VUS in DNAH8 [c.9839A>T (p.Gln3280Leu)]. Familial genetic studies confirmed the maternal inheritance of the RSPH4A [c.1103T >G (p.Val368Gly)] variant. No paternal RSPH4A or DNAH8 variants were detected. As a result of the subsequent familial analysis, reclassification was made for the RSPH4A [c.1103T >G (p.Val368Gly)] variant as pathogenic.

\section{Case 2: RSPH4A homozygous female pediatric case}

A six-year-old native Puerto Rican female presented with a past medical history of daily wet cough, recurrent pulmonary infections, bronchiectasis, sinusitis, and neonatal respiratory distress despite term gestation that required NICU admission for 14 days. Multiple hospitalizations were reported through her first six years of life due to chronic bronchitis and recurrent pneumonias. The patient had a prior surgical history of adenoidectomy at four years old. Physical examination revealed bibasilar crackles, monophonic wheezes, and mild generalized clubbing. Chest imaging showed bilateral opacities concerning for pneumonia, and HRCT of the chest showed bilateral cylindrical bronchiectasis on RML, LLL, and lingula. Ciliary biopsy revealed rare cilia but was not diagnostic for PCD. Spirometry was consistent with air trapping with FVC: 107\%, FEV1: 102\%, FEV1/FVC: 95\%, TLC: 132\%, RV: 244\%, and RV/TLC: 174\% percentage of predicted. Bronchoscopy revealed normal airway anatomy and the presence of bilateral whitish bronchial secretions at the distal airways. Bronchoalveolar lavage fluid was negative for pulmonary infection. A followup outpatient throat culture was positive for Pseudomonas aeruginosa. The sweat test was $15.7 \mathrm{mEq} / \mathrm{L}$ on the right arm and $13.2 \mathrm{mEq} / \mathrm{L}$ on the left arm. Genetic testing results were negative for CFTR and primary immunodeficiencies genes. Serum immunoglobulin titers were normal. The FeNO test was $<5 \mathrm{ppm}$. Measurement of nNO was unavailable. A homozygous pathogenic RSPH4A gene [c.921+3_921+6delAAGT] splice site mutation was identified on subsequent PCD genetic testing. Additionally, a pathogenic variant in DNAH11 [c.3133C > T (p.Arg1045*)] was present. The maternal genetic analysis detected the presence of the RSPH4A mutation but the DNAH11 genetic variant was not detected. Paternal testing was not available.

\section{Methodology and results of the ancestral RSPH4A analysis}

To confirm the RSPH4A [c.921+3_921+6delAAGT] splice site mutation ancestry, the Phylogenetic Network Software of fluxus-engineering.com was used to construct a median-joining haplotype network [13] with genome sequence data from 104 Puerto Rican subjects in the 1000 Genomes Project (1000GP) [14]. The haplotype network (Figure 1 ) was constructed using the 20 polymorphic sites genotyped by 23andme that are located from nucleotide positions $116,864,852$ to $117,021,275$ in chromosome six, according to the GRCh37 human genome assembly. The span starts $72,790 \mathrm{bp}$ upstream of the transcription initiation site of the RSPH4A gene and ends 67,127 bp downstream of its 3' end. 


\section{Cureus}

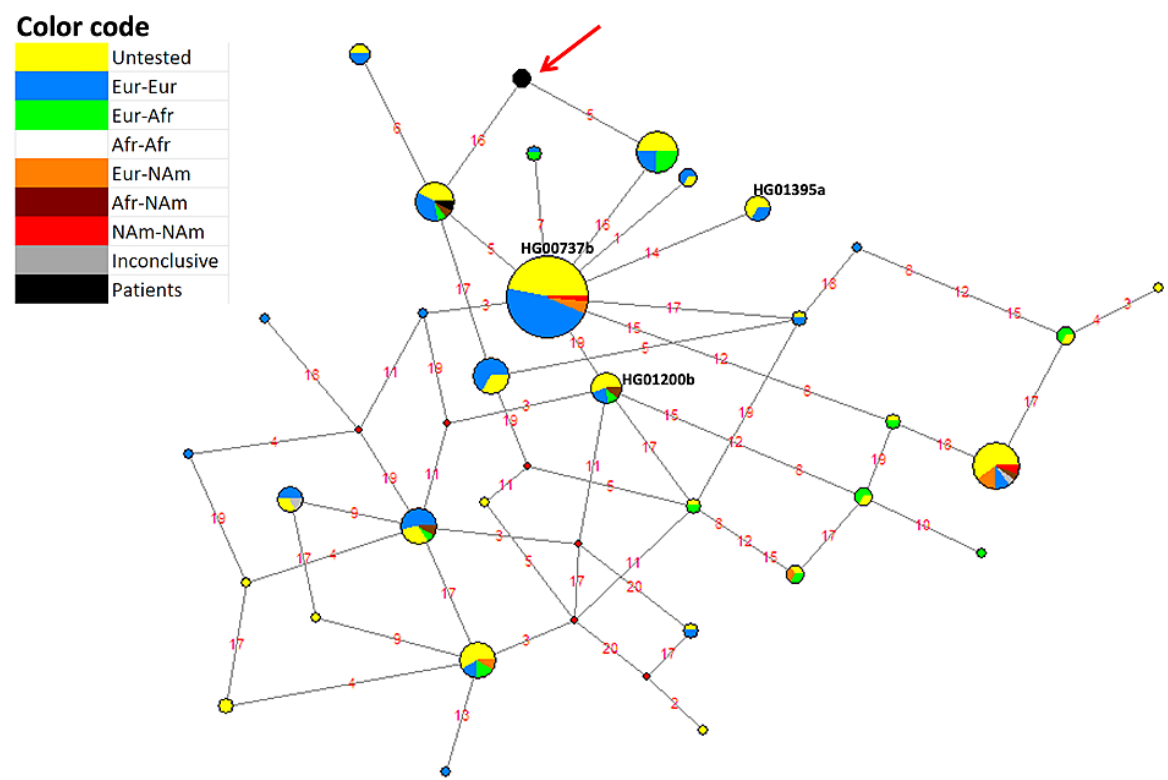

\section{FIGURE 1: Haplotype network of the region spanning from nucleotide positions $116,864,852$ to $117,021,275$ in chromosome six in 104 Puerto Rican subjects in the $1000 \mathrm{GP}$.}

Haplotypes are represented by circles of size proportional to the number of chromosomes they represent. Hypothetical haplotypes are represented by red diamonds. Haplotype colors refer to their predicted ancestry. A haplotype that represents only patient chromosomes is highlighted with a red arrow. Lines connect haplotypes differing by polymorphisms that are represented by red numbers: $1=$ rs2352482, $2=$ rs $117750891,3=r s 17078051,4=r s 76319074,5=r s 7752566,6=r s 11755235,7=r s 62424373,8=$ rs $9481650,9=r s 41289942,10=r s 111375012,11=r s 6927567,12=r s 9488991,13=r s 146142715,14=$ $r s 13202520,15=r s 9488999,16=r s 72959988,17=r s 9489008,18=r s 1165376,19=r s 9481657,20=$ rs72962017. Black numbers next to three haplotypes indicate the haplotypes to which three chromosome copies with the RSPH4A [c.921+3 921+6delAAGT] splice site mutation in the 1000GP belong.

The most common haplotype in Figure 1 consists only of non-African chromosomes, and likely represents an out-of-Africa founder haplotype from which most of the remaining non-African haplotypes arose. One of the 62 chromosome copies that compose this haplotype (HG00737b) possess the RSPH4A [c.921+3 921+6delAAGT] splice site mutation, and two other haplotypes, each differing from the founder haplotype by a single polymorphism, also contain a chromosome copy with the mutation (HG01200b and HG01395a). The 1000GP data estimates the frequency of the RSPH4A [c.921+3 921+6delAAGT] splice site mutation in Puerto Rico at 1.4\% (3/208). It is a low-frequency but not rare allele in Puerto Rico in spite of its pathogenicity. Furthermore, the RSPH4A [c.921+3_921+6delAAGT] splice site mutation seems to have arisen in an out-of-Africa founder haplotype. At least two subsequent mutations, identified in Figure 1 as polymorphisms \#14 and \#19, generated new haplotypes holding the RSPH4A [c.921+3_921+6delAAGT] splice site mutation.

Figure 1 also shows a haplotype that only consists of three patient chromosome copies (red arrow). Two of the three chromosomes belong to a patient homozygous for the RSPH4A [c.921+3_921+6delAAGT] splice site mutation (Case 1), and the other to a compound heterozygous patient holding one copy of the same mutation (Case 2). The remaining chromosome of the latter patient differs only at rs72959988 (SNP \#16 in Figure 1). Thus, it is highly likely that the three patient chromosomes with the RSPH4A [c.921+3_921+6delAAGT] mutation share the same, unique haplotype, which, for simplicity, we will call "the disease haplotype." In conclusion, the data from our patients strongly suggest that, in addition to polymorphisms \#14 and \#19, a third polymorphism arose in the founder haplotype, either polymorphism \#5 or polymorphism \#16, to generate another haplotype holding the RSPH4A [c.921+3_921+6delAAGT] splice site mutation. This third derived haplotype with the RSPH4A [c.921+3_921+6delAAGT] splice site mutation recombined with a haplotype holding either polymorphism $\# 5$ or polymorphism \#16 to generate the disease haplotype, which possesses both polymorphisms \#5 and \#16.

The three mutations occurring after the rise of the RSPH4A [c.921+3_921+6delAAGT] splice site mutation allow us to estimate its time of origin. Applying the human nucleotide nuclear mutation rate of $2.5 \times 10-8$ mutations per nucleotide site to the studied fragment, which is $156,423 \mathrm{bp}$ long, and using 30 years to 
represent generation time [15], we estimate the age of RSPH4A [c.921+3_921+6delAAGT] splice site mutation at 23,015 years. This is approximately 8,000 years prior to human arrival to the New World.

\section{Discussion}

Previous studies have identified the founder mutation RSPH4A [c.921+3_6delAAGT] in the Hispanic Puerto Rican population $[7,10]$. This study explored the ancestry of the founder mutation in two genetically confirmed PCD subjects with genetic mutations in the RSPH4A gene. Determining the origin of a mutation can be a difficult task, especially in a highly admixed population like Puerto Rico. In this study, we base our analysis on three lines of evidence: local ancestry of chromosomal segments carrying the RSPH4A [c.921+3_921+6delAAGT] splice site mutation, the origin of neighboring haplotypes in the haplotype network, and age.

Phase 1 of the 1000GP presented the genome sequence of 55 Puerto Ricans, all of which were subjected to local ancestry analysis [14]. A female of these 55 Puerto Ricans (HG00737) held the RSPH4A

[c.921+3_921+6delAAGT] splice site mutation in its heterozygous form, but both of her chromosomal segments holding the RSPH4A gene were determined by the local ancestry analysis to be of European origin. It is thus likely that the RSPH4A [c.921+3_921+6delAAGT] splice site mutation arrived in Puerto Rico from Europe.

The haplotype of this chromosome segment is a likely founder of the out-of-Africa migration that started populating the rest of the world some 60,000 years before present (YBP). However, we estimate the mutation to have arisen only 23,015 YBP. Hence, the haplotype had plenty of time to spread all over Eurasia before the mutation arose. However, the data suggest that the geographic extension of the mutation is very limited because it only appeared in three Puerto Ricans and not once in any of the other 25 world populations that were sampled by the 1000GP. The mutation is estimated to have arisen before the colonization of the New World from a very small Siberian population approximately 15,000 YBP [16]. Considering the bottleneck effect that occurred during this event, and the fact that the Caribbean was the last region of the Americas to be colonized by humans [17], had the mutation arrived in Puerto Rico from its Native Americans, it would have been reasonable to expect that it must have been present and found in other American populations.

These findings contrast the original hypothesis suggested by Leigh et al., who attributed the origin of the founder mutation to the Taino ancestry due to the presumable prevalence of recessive conditions maternally inherited through mitochondrial genes and favored by the island's geographically isolation [7,12]. Future research could consider the analysis of this dataset with an additional range of chromosomal segments to further explore the origin of the RSPH4A [c.921+3_6delAAGT] splice site mutation, the actual prevalence of the condition on the island, and the applicability of the approaches used to date.

Finally, additional facts supporting the European origin of the founder mutation RSPH4A [c.921+3_6delAAGT] are: first, the mutation has a single origin implied from the type of mutation, and the 1000 GP Database identified unambiguously in the HG00737b mutation is from European origin. Hence, the two patient's chromosomes have a single origin, and one is strictly European. Furthermore, haplotype HG01395a, which has the mutation of interest, only contains chromosomes of European origin.

\section{Conclusions}

One of the conclusions of the 1000GP was that deleterious mutations were commonly limited to single populations. As the age of RSPH4A [c.921+3_921+6delAAGT] bars the possibility that it arose in Puerto Rico after its colonization by Europeans, it is likely that its origin arose from a limited region within Spain. The diversity of haplotypes holding this mutation strongly suggests that the region was a big source of colonists who brought such haplotypes to Puerto Rico in early colonial times. The haplotypes quickly increased in frequency due to the genetic drift and the population expansion that followed. As a conclusion, the ancestral haplotype analysis presented shows that chromosome segments with haplotypes equal to those of the chromosomes carrying the RSPH4A [c.921+3_6delAAGT] splice site mutation had predominantly European ancestry.

\section{Additional Information \\ Disclosures}

Human subjects: Consent was obtained or waived by all participants in this study. University of Puerto Rico, Medical Sciences Campus, Department of Pediatrics issued approval B1730120. Institutional Review Board of the University of Puerto Rico, Medical Sciences Campus, Department of Pediatrics issued approval B1730120. The study was conducted according to the guidelines of the University of Puerto Rico, Medical Sciences Campus, and approved by the Institutional Review Board of the University of Puerto Rico, Medical Sciences Campus, Department of Pediatrics. Conflicts of interest: In compliance with the ICMJE uniform disclosure form, all authors declare the following: Payment/services info: This research was partially funded by: Hispanic Center of Excellence, University of Puerto Rico School of Medicine (Grant Number: D34HP24463), U.S. Department of Health and Human Services, Health Resources and Services Administration, Bureau of Health Workforce. UPRMSC Hispanics-In-Research Capability (HiREC) 
Endowment, Partnership between the School of Health Professions and School of Medicine, University of Puerto Rico, Funded (S21MD001830) by the NIH National Institute of Minority Health and Health Disparities. The National Institute of Health: Award Number: HCTRECD R25MD007607 from the National Institute on Minority Health and Health Disparities. The content is solely the responsibility of the authors and does not necessarily represent the official views of the National Institutes of Health. Financial relationships: All authors have declared that they have no financial relationships at present or within the previous three years with any organizations that might have an interest in the submitted work. Other relationships: All authors have declared that there are no other relationships or activities that could appear to have influenced the submitted work.

\section{References}

1. Knowles MR, Daniels LA, Davis SD, Zariwala MA, Leigh MW: Primary ciliary dyskinesia. Recent advances in diagnostics, genetics, and characterization of clinical disease. Am J Respir Crit Care Med. 2013, 188:913-22. 10.1164/rccm.201301-0059CI

2. Leigh MW, Horani A, Kinghorn B, O'Connor MG, Zariwala MA, Knowles MR: Primary ciliary dyskinesia (PCD): a genetic disorder of motile cilia. Transl Sci Rare Dis. 2019, 4:51-75.

3. O'Connor MG, Horani A, Shapiro AJ: Progress in diagnosing primary ciliary dyskinesia: the North American perspective. Diagnostics (Basel). 2021, 11:1278. 10.3390/diagnostics11071278

4. Zariwala MA, Knowles MR, Leigh MW: Primary Ciliary Dyskinesia. GeneReviews ${ }^{\circledR}$. Adam MP, Ardinger HH, Pagon RA, Wallace SE, Bean LJH, Mirzaa G, Amemiya A (ed): University of Washington, Seattle; 2007.

5. O'Connor MG, Griffiths A, Iyer NP, Shapiro AJ, Wilson KC, Thomson CC: Summary for clinicians: diagnosis of primary ciliary dyskinesia. Ann Am Thorac Soc. 2019, 16:171-4. 10.1513/AnnalsATS.201810-693CME

6. Shapiro AJ, Davis SD, Polineni D, et al.: Diagnosis of primary ciliary dyskinesia. An official American Thoracic Society clinical practice guideline. Am J Respir Crit Care Med. 2018, 197:e24-39.

10.1164/rccm.201805-0819ST

7. Daniels ML, Leigh MW, Davis SD, et al.: Founder mutation in RSPH4A identified in patients of Hispanic descent with primary ciliary dyskinesia. Hum Mutat. 2013, 34:1352-6. 10.1002/humu.22371

8. Shapiro AJ, Leigh MW: Value of transmission electron microscopy for primary ciliary dyskinesia diagnosis in the era of molecular medicine: genetic defects with normal and non-diagnostic ciliary ultrastructure. Ultrastruct Pathol. 2017, 41:373-85. 10.1080/01913123.2017.1362088

9. Castleman VH, Romio L, Chodhari R, et al.: Mutations in radial spoke head protein genes RSPH9 and RSPH4A cause primary ciliary dyskinesia with central-microtubular-pair abnormalities. Am J Hum Genet. 2009, 84:197-209. 10.1016/j.ajhg.2009.01.011

10. De Jesús-Rojas W, Reyes-De Jesús D, Mosquera RA: Primary ciliary dyskinesia diagnostic challenges: understanding the clinical phenotype of the Puerto Rican RSPH4A founder mutation. Diagnostics (Basel). 2021, 11:281. 10.3390/diagnostics11020281

11. Via M, Gignoux CR, Roth LA, et al.: History shaped the geographic distribution of genomic admixture on the island of Puerto Rico. PLoS One. 2011, 6:e16513. 10.1371/journal.pone.0016513

12. Martínez-Cruzado JC, Toro-Labrador G, Ho-Fung V, et al.: Mitochondrial DNA analysis reveals substantial Native American ancestry in Puerto Rico. Hum Biol. 2001, 73:491-511. 10.1353/hub.2001.0056

13. Bandelt HJ, Forster P, Röhl A: Median-joining networks for inferring intraspecific phylogenies . Mol Biol Evol. 1999, 16:37-48. 10.1093/oxfordjournals.molbev.a026036

14. Auton A, Brooks LD, Durbin RM, et al.: A global reference for human genetic variation . Nature. 2015, 526:68-74. 10.1038/nature15393

15. Tremblay M, Vézina H: New estimates of intergenerational time intervals for the calculation of age and origins of mutations. Am J Hum Genet. 2000, 66:651-8. 10.1086/302770

16. Fagundes NJ, Kanitz R, Eckert R, et al.: Mitochondrial population genomics supports a single pre-Clovis origin with a coastal route for the peopling of the Americas. Am J Hum Genet. 2008, 82:583-92. 10.1016/j.ajhg.2007.11.013

17. Moreno-Estrada A, Gravel S, Zakharia F, et al.: Reconstructing the population genetic history of the Caribbean. PLoS Genet. 2013, 9:e1003925. 10.1371/journal.pgen.1003925 